\title{
Utilization Rates of Implantable Cardioverter-Defibrillators for Primary Prevention of Sudden Cardiac Death: A 2012 Calculation for a Midwestern Health Referral Region
}

\author{
Allen Hoang, $\mathrm{MD}^{1}$, Changyu Shen, $\mathrm{PhD}^{2,5}$, James Zheng, $\mathrm{PhD}, \mathrm{MHA}^{3}$, Stanley Taylor, $\mathrm{MA}^{2}$, \\ William J Groh, MD, MPH, FHRS ${ }^{1}$, Marc Rosenman, MD ${ }^{4}$, Alfred E. Buxton, MD ${ }^{6}$, and Peng- \\ Sheng Chen, MD, FHRS ${ }^{1}$ \\ ${ }^{1}$ Krannert Institute of Cardiology and the Division of Cardiology, Indiana University, Indianapolis, \\ IN
}

${ }^{2}$ Department of Biostatistics, Indiana University, Indianapolis, IN

${ }^{3}$ The Regenstrief Institute, Indiana University, Indianapolis, IN

${ }^{4}$ Department of Pediatrics, Indiana University, Indianapolis, IN

${ }^{5}$ School of Public Health, Indiana University, Indianapolis, IN

${ }^{6}$ Cardiovascular Division, Department of Medicine, Beth Israel Deaconess Medical Center and Harvard Medical School, Boston, MA

\begin{abstract}
Background-Utilization rates (URs) for implantable cardioverter-defibrillators (ICDs) for primary prevention of sudden cardiac death (PPSCD) are lacking in the community.

Objective-To establish the ICD UR in central Indiana.

Methods-A query run on two hospitals in a health information exchange database in Indianapolis identified patients between 2011 and 2012 with left ventricular ejection fraction (EF) \$).35. ICD-eligibility and utilization were determined from chart review.

Results-We identified 1,863 patients with at least one low-EF study. Two cohorts were analyzed: 1,672 patients without, and 191 patients with, ICD-9-CM procedure code 37.94 for ICD placement. We manually reviewed a stratified (by hospital) random sample of 300 patients from
\end{abstract}

\footnotetext{
(C) 2014 The Heart Rhythm Society. Published by Elsevier Inc. All rights reserved.

Address for Correspondence: Peng-Sheng Chen, MD, 1800 N. Capitol Ave, E 475, Indianapolis, IN 46202, Phone: 317-962-0145 / Fax: 317-962-0588 / chenpp@iupui.edu.

Disclosures

Dr. Buxton: served on clinical trial event committees of studies by Medtronic, Inc., St. Jude Medical, Boston Scientific Corp, has received travel support from Medtronic. His institution received research grants from Medtronic and Biosense-Webster. His institution receives fellowship support from Medtronic, Boston Scientific, and Biosense-Webster; Dr. Chen's research laboratory has equipment donated by Cyberonics, Inc, Medtronic, Inc, and St. Jude Medical, Inc.

Publisher's Disclaimer: This is a PDF file of an unedited manuscript that has been accepted for publication. As a service to our customers we are providing this early version of the manuscript. The manuscript will undergo copyediting, typesetting, and review of the resulting proof before it is published in its final citable form. Please note that during the production process errors may be discovered which could affect the content, and all legal disclaimers that apply to the journal pertain.
} 
the no-ICD procedure code cohort and found that $48(16 \%)$ had no ICD but had class I indications for ICD. Eight of 300 (2.7\%) actually had ICD implantation for PPSCD. Review of all 191 patients in the ICD procedure code cohort identified 70 with ICD implantation for PPSCD. The ICD UR (ratio between patients with ICD for PPSCD and all with indication) was $38 \%$ overall (95\% CI 28-49\%). URs were $48 \%$ for males (95\% CI 34-61\%), $21 \%$ for females (95\% CI 16$26 \%, \mathrm{p}=0.0002$ vs males), $40 \%$ for whites ( $95 \%$ CI $27-53 \%$ ), and $37 \%$ for blacks (95\% CI 28 $46 \%, \mathrm{p}=0.66$ vs whites).

Conclusions-The ICD UR is $38 \%$ among patients meeting Class I indications, suggesting further opportunities to improve guideline compliance. Furthermore, this study illustrates limitations in calculating ICD UR using large electronic repositories without hands-on chart review.

\section{Keywords}

defibrillator; implantable cardioverter-defibrillator; sudden cardiac death; arrhythmia; utilization

\section{INTRODUCTION}

Multiple studies have demonstrated a mortality benefit of implantable cardioverterdefibrillators (ICDs) in the primary prevention of sudden cardiac death (PPSCD) in selected patients with left ventricular dysfunction. ${ }^{1,2,3,4}$ This led to the adoption of ICD therapy for PPSCD and a Class IA recommendation for cardiology practice. ${ }^{5-7}$ However, not all eligible patients receive an ICD. Shah et al, using The Get With The Guidelines-Heart Failure (GWTG-HF) registry, described ICD utilization rates (URs) from hospitals across the United States. ${ }^{8}$ With rigorous methods of analyses, the GWTG-HF URs were found to be as low as $1 \%$ in the lowest tertile of hospitals to as high as $80 \%$ in the upper tertile. The region with most hospitals in the upper tertile of ICD utilization was the Midwest. ${ }^{8}$ The URs were not reported specifically for each geographic region, so the ICD UR in the community remains unclear. Other studies assessing (PPSCD) ICD URs confirm the low rates and high variability, as low as $28 \%$ and as high as $80 \%^{10}$ in the US. These URs reflect University tertiary care teaching hospitals ${ }^{11,12}$ and Veterans Affairs Hospitals, ${ }^{9,} 10$ but none mentioned the inclusion of safety-net hospitals, where a more representative assessment of the community's adherence to guideline-based therapy can be made. Furthermore, few of these studies were derived from comprehensive chart review. Because of these limitations, the ICD UR in the community remains unclear. Other notable findings from literature review show that women are less likely than men to receive ICDs, ${ }^{13-16}$ that blacks are less likely than whites to receive ICDs, ${ }^{14,} 17,18$ and there is great disparity in ICD implantation rates among hospital referral regions in the US. ${ }^{19,} 20$ We sought to establish the ICD UR in central Indiana, by collecting data from a not-for-profit, University-affiliated tertiary care teaching hospital and a county hospital and reviewing the patient charts to more accurately reflect the real-world community.

\section{METHODS}

Because chart review validation would be integral to this study, we selected two hospitals for which access to all medical records (including text reports) was readily available. These 
two hospitals account for $44 \%$ of the inpatient beds among the six major hospitals in the area. All six of the major hospitals have subspecialty cardiology services. ${ }^{21}$ We sought to ascertain the ICD UR over a recent twelve-month period (6/1/2011-5/31/12), through database query and chart review. The study was approved by the institutional review board of Indiana University and Purdue University Indianapolis.

The UR is defined in Equation 1. The strategy to identify patients in both $x$ and $y$ is outlined below.

Equation 1. Utilization Rate (UR)

$$
U R(\%)=\frac{x}{x+y} \times 100 \%
$$

Where $x=$ number of patients with ICD's implanted for PPSCD during a selected period ("ICD-utilized") and $y=$ number of patients with indication for ICD therapy but not implanted during the study period ("ICD-eligible").

\section{Low Ejection Fraction Query}

We first identified a cohort of patients with at least one left ventricular ejection fraction (EF) $\$ 0.35$ by echocardiography between June 1, 2011 and May 31, 2012. Data from the health information exchange were extracted via Structured Query Language. Service codes (medical record dictionary terms) for echocardiograms were used to query EF values either directly or by calculation from left ventricular diastolic volume and systolic volume. A patient with at least one qualifying $\mathrm{EF}$ value obtained through either method was included in the low EF cohort. To identify low-EF patients who received an ICD during the study period, we used billing codes for ICD implantations. From the International Classification of Diseases, Ninth Revision, Clinical Modification (ICD-9-CM), the inpatient hospital procedure code 37.94 (Implantation or replacement of automatic cardioverter/defibrillator, total system [AICD]) was examined in our first query. We also searched for the code V45.02 (Automatic implantable cardiac defibrillator in situ) to identify patients with previous ICD implantation. Current Procedural Terminology (CPT) Code 33249 (Insertion or repositioning of electrode lead(s), for single or dual chamber pacing cardioverter defibrillator and insertion of pulse generator) was also used in an attempt to further screen for ICD implantations.

\section{Chart Review}

From the low-EF cohort generated by the automated query, those without code 37.94 were defined as Cohort A, and those with the code as Cohort B (Figure 1). Three hundred patients were randomly selected (150 from each hospital) from Cohort A for chart review, and from Cohort B, all 191 (total of 491). Patients' medical record numbers (MRNs) were randomly assigned a study number based on hospital. A standardized chart abstraction method was applied to promote consistency, recording de-identified patient data into database software $\left(\operatorname{RedCap}^{\mathrm{TM}}\right)$. 
In all 491 charts reviewed, echocardiogram reports were scrutinized to find EFs recorded by physicians (as opposed to EFs extracted by the automated query, which reflect calculated values based on measurements entered by echocardiogram technicians). The reviewer used the clinician's stated EF in the official report to determine eligibility for ICD. Diagnostic reports, including echocardiograms, ventriculograms, and radionuclide imaging (including those not pre-populated by the automated low EF query) were reviewed to confirm EF.

Review for ICD Indication-Once patients with low EF were identified, defined as EF $\$$ ).35, a review for ICD implantation was carried out as described in the sections below. Those without ICD were considered as candidates for ICD therapy by having EF $₫ 0.35$ alone, without respect to ischemic disease, unless there were contraindications for ICD based on the 2008 American College of Cardiology/American Heart Association/Heart Rhythm Society (ACC/AHA/HRS) Guidelines for Device-Based Therapy of Cardiac Rhythm Abnormalities, ${ }^{7}$ or obvious reasons why ICD would not be standard of care (Figure 1). These guidelines state that ICD is indicated in patients with LV dysfunction due to prior MI who are at least 40 days post-MI, have an EF less than $30 \%$ with NYHA functional Class I or EF less than 35\% with NYHA II or III. However, because NYHA functional class and ischemic disease could not be confirmed across all charts, the primary inclusion criterion was EF $₫ \mathbf{0 . 3 5}$.

Verification of Utilized ICD - Cohort A-From Cohort A, ICD-implanted patients who had not been identified by code 37.94 underwent chart review to exclude implantations before the study period (6/1/2011), non-low EF, or non-primary prevention indications (Figure 1). These patients had either code V45.02 or CPT code 33249. Patients receiving cardiac resynchronization therapy (CRT) cardioverter-defibrillators (CRT-D) were treated in the same way as non-CRT ICDs by reviewing the indication for SCD prevention.

Verification of Utilized ICD - Cohort B-Among the 191 patients in Cohort B (all with code 37.94), manual chart review was performed in the same way as for those with ICD implants in Cohort A above (Figure 1). Implantations from both cohorts thus comprised the number of ICD's implanted for PPSCD during the study period.

\section{Utilization Rate Calculation}

The UR is calculated based on Equation 1, of which Equation 2 is a modification, including variables specific to the study design.

Equation 2. ICD Utilization Rate, expanded

$$
U R(\%)=\frac{C x_{A}+x_{B}}{\left(C x_{A}+x_{B}\right)+D y_{A}+y_{B}} \times 100 \%
$$

Where

$C, D=$ sampling correction factors for Cohort A

$x_{A}=$ patients with ICD indication and implantation in samples of Cohort A 


$$
\begin{aligned}
& x_{B}=\text { patients with ICD indication and implantation, Cohort B } \\
& y_{A}=\text { patients with ICD indication but without implantation in samples of Cohort A } \\
& y_{B}=\text { patients with ICD indication but without implantation, Cohort B }
\end{aligned}
$$

Cohort A is composed of an unequal number of patients from two hospitals, from which the same number of patients were sampled (150 from each). Therefore, correction factors were used to account for the disproportional sampling rate as outlined in Equation 3. The numerator in Equation 2 estimates the number of patients with ICD among the 1,863 low EF subjects, and the denominator estimates the number of patients eligible for primary ICD, where $C x_{A}$ and $D y_{A}$ represent the estimated numbers of eligible patients with and without ICD in Cohort A of 1,672 subjects. Of note, $y_{B}$ is a theoretical population and was not encountered in the present study, i.e. no patient with code 37.94 lacked an ICD and met low EF criteria for PPSCD.

Equation 3. Sampling correction for Cohort A

$$
\begin{aligned}
& C x_{A}=C_{1} x_{1 a}+C_{2} x_{2 A} \\
& D y_{A}=D_{1} y_{1 a}+D_{2} y_{2 A}
\end{aligned}
$$

Where

$C_{1}, D_{1}=$ sampling correction factors for Hospital 1

$C_{2}, D_{2}=$ sampling correction factors for Hospital 2

$x_{1 A}, x_{2 A}$ : patients with ICD indication and implantation in samples of each of the two hospitals from Cohort A

$y_{1 A}, y_{2 A}$ : patients with ICD indication but without implantation in samples of each of the two hospitals from Cohort A

Because there are 1,267 and 405 patients from the two hospitals in Cohort A, a sample of 150 from each represents a sampling rate of 1 in 8.45 and 1 in 2.7, respectively. Therefore, $C_{1}$ and $D_{1}=8.45, C_{2}$ and $D_{2}=2.7$, and $C x_{A}$ and $D y_{A}$ hence represent the projected number of patients with and without ICD in Cohort A of 1,672 subjects. Confidence intervals of the UR are computed based on normal approximation that accounts for the sampling corrections.

\section{RESULTS}

\section{Low-EF Query}

We searched the electronic medical records of 4,352,020 patients and identified a total of 21,663 unique patients who had an EF documented between 6/1/2011 and 5/31/2012. From this pool, 1,863 patients were identified as having EF $₫) .35$ at least once (Figure 1). 


\section{Utilized ICDs $\left(C x_{A}\right.$ and $\left.x_{B}\right)$}

Cohort A - Calculation of $\mathbf{C x}_{\mathbf{A}}$ - In Cohort A, there were a total of 1,672 patients without ICD-9-CM code 37.94. Thirty-four of the sampled 300 patients had an ICD (despite absence of code 37.94) (Figure 2). Twenty-four of 34 had an ICD coded under ICD-9-CM diagnosis code V45.02, which suggests that the devices were implanted at a hospital outside the two hospitals in the study or were erroneously not assigned code 37.94. The remaining 10 of 33 carried none of the three codes 37.94 , V45.02, or 33249. A query confirmed that patients with CPT code 33249 were completely accounted for by queries for 37.94 or V45.02; therefore, CPT code 33249 was not required to further identify patients with ICD devices.

By indication, 8 of the 33 were new implants for PPSCD associated with physicianconfirmed low EF (Figure 2). The other 26 patients with an ICD had these four scenarios (multiple qualifications were assigned with descending priority, i.e. 1>2>3): (1) ICD implanted before the 12-month period [ $\mathrm{N}=22]$ or unavailable confirmed date of original implant [N=1], (2) ICD for secondary prevention [N=1], or (3) ICD implanted despite last $\mathrm{EF}>0.35$ [N=1]. The 8 patients with new implants for PPSCD associated with physicianconfirmed low EF implies an estimated number of $50\left(c x_{A}\right)$ over Cohort A based on Equation 3.

Cohort B - Calculation of $\boldsymbol{x}_{\boldsymbol{B}}$-There were a total of 191 patients among the low-EF query whose charts contained procedure code 37.94 , of which 164 had ICD implantation within the selected timeframe (Figure 3). The rest were excluded: 26 with ICD implantation before the 12-month study period and 1 incorrectly with a 37.94 code for a dual-chamber pacemaker without defibrillator function. By indication, 88 were excluded due to secondary prevention indication, 4 excluded for long QT syndrome or hypertrophic cardiomyopathy, and 2 excluded for primary prevention with EF $>0.35$. The remainder, $70\left(x_{B}\right)$, of the 191 patients in Cohort B had a new primary prevention ICD implantation during the study period and physician-confirmed low EF (Figure 3).

\section{ICD-Eligible $\left(D y_{A}\right.$ and $\left.y_{B}\right)$}

In the review of 300 patients from Cohort A, 137 patients without ICD were confirmed by the chart review to actually have EF $₫ 0.35$ (Figure 2). Excluded were patients with low EF as detected by query (based on sonographer measurements) but whose official EF as read by the cardiologist (which is not searchable by query) was $>0.35$. Patients were also excluded if a follow-up EF assessment showed EF $>0.35$. These exclusions totaled 127. One patient had an inconclusive $\mathrm{EF}$, and one record was a duplicate of a patient who was confirmed as low EF (Figure 2). Of the 137 with a confirmed low EF, 48 were ICD-eligible, i.e. having no contraindications (Figure 2). According to Equation 3, there were an estimated 193 patients $\left(D y_{A}\right)$-over 1,672 in Cohort A—who met indications for ICD implantation for PPSCD but did not have an ICD. Zero such patients from Cohort B were identified $\left(y_{B}=0\right)$.

\section{Utilization Rate Calculation}

Given the variables determined above $-C x_{A}, x_{B}, C y_{A}$, (and $y_{B}$ which was zero) - for ICDutilized and ICD-eligible without ICD during the study period, the UR could then be 
calculated according to Equation 2. The ICD UR was 38\% (120/313, 95\% CI 28-49\%). Utilization rates were $48 \%$ for males (95\% CI 34-61\%), $21 \%$ for females (95\% CI 16-26\%, $\mathrm{p}=0.0002$ vs males), $40 \%$ for whites (95\% CI $27-53 \%$ ), and $37 \%$ for blacks (95\% CI 28 $46 \%, \mathrm{p}=0.66$ vs whites). The mean age of patients with an ICD was 55.7 years (57.0 median, interquartile range 42.4-67.8 years), and of patients without ICD, mean 60.9 years (63.3 median, interquartile range 52.7-66.5) $(\mathrm{p}=0.11)$.

\section{Reasons for No ICD}

The review of 300 patients from Cohort A revealed 137 with confirmed low EF (Table 1). Forty-eight of 137 had no contraindications to ICD and were included in the UR calculation above. Among the remaining 89, the contraindications included the following: heart failure diagnosed within 3 months of low EF (11\% of Cohort A patients with low EF), death before work-up (11\%), MI within 40 days or percutaneous coronary intervention/ coronary artery bypass grafting (PCI/CABG) within 3 months (11\%), patient refusal (9\%), do not resuscitate status (DNR) (8\%), active and advanced cancer (6\%), non-compliance with medical or dietary therapy (4\%), dementia (4\%), functional NYHA Class I non-ischemic heart failure $(3 \%)$, awaiting kidney transplant (1\%), primary care physician refusal (1\%), pregnancy $(<1 \%)$, or lost to follow-up after ICD recommendation $(<1 \%)$.

For the 48 patients considered ICD-eligible but without ICD, a separate review was carried out in December 2013 to elucidate the extent to which these patients were evaluated for ICD indication. Among the 48 initially with no apparent contraindications, ICD was mentioned in inpatient or outpatient notes for 12 patients. Among these 12, ICD was discussed in greater detail in 6 cases, 3 of whom were offered ICD. Two of the 3 patients refused, while one was deferred by the cardiologist due to emaciation. For 36 patients, ICD was never mentioned at the time of the low EF study or the immediate follow-up visit. Among these, 6 patients were later found to have been awaiting revascularization or valve surgery.

\section{DISCUSSION}

\section{Previously Calculated ICD Utilization Rates}

The reported ICD UR among the eligible populations has been highly variable. At a Veterans Affairs Medical Center (VAMC) in California, the ICD UR was found to be $28 \%$ from 2002 to $2006 .{ }^{9}$ Atwater et al ${ }^{10}$ reported an ICD UR of $80 \%$ in 2010 at a VAMC in North Carolina. Both studies used chart review and assessed for contraindications to ICD, which would mitigate a falsely low UR. Great disparity in ICD implantation rates among hospital referral regions in the United States has been described in the literature, ${ }^{22}$ therefore, caution is advised when comparing ICD URs from those locales and from those time periods.

While we found that the ICD UR for PPSCD is low, the ICD UR for secondary prevention was also reported as low $(<40 \%)$ in a database study ${ }^{23}$ and a chart review. ${ }^{24}$ The low rate of guideline compliance is not limited to device therapy, as $25 \%$ of the patients with an ICD did not receive optimal medical therapy in one study. ${ }^{25}$ 
The most comprehensive assessment of ICD URs in the country to date was reported by Shah et al in 2009, based on data gathered from the GWTG-HF quality improvement program. ${ }^{8}$ ICD URs were reported to be $17 \%$ overall, with rates ranging from $1 \%$ to $80 \%$. The present study offers a different perspective from that of GWTG-HF by focusing on the rates in the community. In addition, because our study was performed several years later than that of GWTG-HF, the time difference may in part explain the different ICD URs between our study and the GWTG-HF.

\section{Hospital Representation of ICD UR in the Region}

The hospitals included in this study comprised $44 \%$ of the Indianapolis metropolitan area's inpatient beds. Logistical reasons in designing the study prevented further involvement of the other systems. However, we feel that having representation from the region's county hospital helps to more accurately portray utilization rates in the community, when combining data with a large private hospital with university affiliation. A true ICD UR in the region is likely to be higher than that reported in this study, because the other (excluded) hospitals include one private hospital with teaching affiliation, two private not-for-profit hospitals, and a VAMC (the latter has 5\% of the inpatient beds in the area). All six of these hospitals provide cardiology subspecialty services. ${ }^{21}$

\section{Benefit of Chart Review}

Chart review is essential for UR accuracy. Many patients who were initially identified as having a low EF were actually found to have $\mathrm{EF}>0.35$ when the cardiologist's interpretation of the echocardiogram was reviewed. Another factor that could skew results without chart review is improvement of EF over time. Our initial query captured patients based on a single low EF. Chart review was required to identify those whose subsequent studies, including non-echocardiogram LV assessment methods, no longer had EF $₫$ ).35. Furthermore, chart review is important because of the number and subjectivity of contraindications.

Particularly, the contraindication "any disease, other than cardiac disease (e.g., cancer, uremia, liver failure), associated with a likelihood of survival less than 1 year" as phrased by the Centers for Medicare \& Medicaid Services decision memorandum ${ }^{26}$ may be inaccurately discerned if one is using diagnostic codes without chart review. Information such as DNR status, patient refusal, and advanced cancer diagnoses, etc., is not conducive to identification via queries.

\section{Reasons for Low ICD Utilization Rate}

Among the 48 ICD-eligible patients without ICD, 36 did not have mention of ICD in the records at discharge or during outpatient follow-up. This implies that physicians who ordered the imaging studies did not appreciate the importance of ICD implantation in patients with a severely reduced EF. Such lack of awareness would prevent a provider from making the appropriate referral for ICD workup. Other provider-based reasons include ineffective dissemination of current practice guidelines, ${ }^{27}$ perceived marginal cost-benefit profile of ICD, and a long period of time required for medical optimization. Patient-driven reasons include not understanding the risk of sudden cardiac death, not following up with Cardiology or Electrophysiology referrals, and/or suboptimal medication adherence leading to delay in evaluation for ICD. As in previous studies, we found that the ICD UR is lower in 
women than in men. However, there is no evidence that UR is different between whites and blacks. The reasons for underutilization of ICD in women remain unclear.

\title{
Proposed Interventions to Improve ICD URs
}

To improve patient understanding of sudden cardiac death risk, more counseling should be given when a diagnostic study reveals EF $₫ 0.35$. A discussion led by the physician with incorporation of nursing education could reinforce a patient's grasp of sudden cardiac death risk. Physician education, likewise, may help improve understanding of ICD benefit and thereby may increase referrals when appropriate. The best place for this discussion should be a clinic setting because of the complexity of indications and the nuances of living with an ICD. To get patients there, an electronic medical record prompt for patients with qualifying low EF at the time of hospital discharge would increase referral rates. Further prompts should follow the initial reminder to reduce unnecessary referrals for those likely to be poor ICD candidates. Furthermore, ongoing risk stratification studies of ICD therapy should improve the ability to identify high-risk patients and thereby increase those patients' likelihood for ICD referral.

\section{Study Limitations}

The sampling correction required to account for Cohort A was necessary because of disproportionate sampling: We reviewed 150 charts from each hospital, but the total of 1,672 patients with low EF in the initial query was not evenly distributed across the two hospitals. Ideally, chart review on all 1,672 patients from Cohort A should be done but could not be done due to resource limitations. Additionally, a likely reason for an inaccurate ICD UR is that patients are lost to follow-up. The present study allows follow-up only within the two hospitals; it misses patients who moved outside of the network during the study period. Furthermore, patients lost to follow-up but remaining in the region may be more likely to refuse ICD therapy. Because refusing ICD therapy is considered a contraindication for the purpose of this study, the presence of such a group would raise the ICD UR by reducing the denominator.

\section{CONCLUSION}

The ICD UR is $38 \%$ among patients meeting Class I indications, suggesting further opportunities to improve guideline compliance. This finding represents one of several areas in heart failure with suboptimal guideline adherence rates. This study illustrates the benefit of hands-on chart review in calculating ICD UR as compared to using only large electronic repositories. Furthermore, this study is the first to combine patients from a private and a county hospital in the same region to provide a more accurate ICD UR for the community.

\section{Acknowledgments}

\author{
Sources of Funding \\ This study was supported in part by NIH Grants P01 HL78931, R0171140, a Medtronic-Zipes Endowment and the \\ Indiana University Health-Indiana University School of Medicine Strategic Research Initiative. \\ We thank Asia Evans, for assistance in interpretation of procedural and diagnostic coding.
}




\section{List of Abbreviations}

ACC

AHA

CABG

CPT

CRT

CRT-D

DNR

EF

GWTG-HF

HRS

ICD

ICD-9-CM

LV

MI

MRN

NYHA

PCI

PPSCD

UR
American College of Cardiology

American Heart Association

coronary artery bypass grafting

Current Procedural Terminology

cardiac resynchronization therapy

cardiac resynchronization therapy cardioverter-defibrillator

do not resuscitate

ejection fraction

Get With Guidelines-Heart Failure (GWTG-HF) registry

Heart Rhythm Society

implantable cardioverter-defibrillator

International Classification of Diseases, Ninth Revision, Clinical

Modification

left ventricular

myocardial infarction

medical record number

New York Heart Association

percutaneous coronary intervention

primary prevention of sudden cardiac death

utilization rate

\section{References}

1. Buxton AE, Lee KL, Fisher JD, Josephson ME, Prystowsky EN, Hafley G. A randomized study of the prevention of sudden death in patients with coronary artery disease. Multicenter Unsustained Tachycardia Trial Investigators. N Engl J Med. 1999; 341:1882-1890. [PubMed: 10601507]

2. Moss AJ, Zareba W, Hall WJ, Klein H, Wilber DJ, Cannom DS, Daubert JP, Higgins SL, Brown MW, Andrews ML. Prophylactic implantation of a defibrillator in patients with myocardial infarction and reduced ejection fraction. N Engl J Med. 2002; 346:877-883. [PubMed: 11907286]

3. Bardy GH, Lee KL, Mark DB, et al. Amiodarone or an implantable cardioverter-defibrillator for congestive heart failure. N Engl J Med. 2005; 352:225-237. [PubMed: 15659722]

4. Kadish A, Dyer A, Daubert JP, et al. Prophylactic defibrillator implantation in patients with nonischemic dilated cardiomyopathy. N Engl J Med. 2004; 350:2151-2158. [PubMed: 15152060]

5. Gregoratos G, Abrams J, Epstein AE, et al. ACC/AHA/NASPE 2002 guideline update for implantation of cardiac pacemakers and antiarrhythmia devices: summary article: a report of the American College of Cardiology/American Heart Association Task Force on Practice Guidelines (ACC/AHA/NASPE Committee to Update the 1998 Pacemaker Guidelines). Circulation. 2002; 106:2145-2161. [PubMed: 12379588]

6. Zipes DP, Camm AJ, Borggrefe M, et al. ACC/AHA/ESC 2006 guidelines for management of patients with ventricular arrhythmias and the prevention of sudden cardiac death: a report of the 
American College of Cardiology/American Heart Association Task Force and the European Society of Cardiology Committee for Practice Guidelines (Writing Committee to Develop Guidelines for Management of Patients With Ventricular Arrhythmias and the Prevention of Sudden Cardiac Death). J Am Coll Cardiol. 2006; 48:e247-e346. [PubMed: 16949478]

7. Epstein AE, Dimarco JP, Ellenbogen KA, et al. ACC/AHA/HRS 2008 guidelines for Device-Based Therapy of Cardiac Rhythm Abnormalities: executive summary. Heart Rhythm. 2008; 5:934-955. [PubMed: 18534377]

8. Shah B, Hernandez AF, Liang L, Al-Khatib SM, Yancy CW, Fonarow GC, Peterson ED. Hospital variation and characteristics of implantable cardioverter-defibrillator use in patients with heart failure: data from the GWTG-HF (Get With The Guidelines-Heart Failure) registry. J Am Coll Cardiol. 2009; 53:416-422. [PubMed: 19179199]

9. Bradfield J, Warner A, Bersohn MM. Low referral rate for prophylactic implantation of cardioverter-defibrillators in a tertiary care medical center. Pacing Clin Electrophysiol. 2009; 32 (Suppl 1):S194-197. [PubMed: 19250092]

10. Atwater BD, Dai D, Allen-Lapointe NM, Al-Khatib SM, Zimmer LO, Sanders GD, Peterson ED. Is heart failure guideline adherence being underestimated? The impact of therapeutic contraindications. Am Heart J. 2012; 164:750-755. e751. [PubMed: 23137506]

11. Chae SH, Koelling TM. Patient and physician determinants of implantable cardioverter defibrillator use in the heart failure population. Congest Heart Fail. 2010; 16:141-146. [PubMed: 20662865]

12. Sims DB, Garcia LI, Mignatti A, Colombo PC, Jones M, Uriel N, Mancini DM, Jorde UP. Utilization of defibrillators and resynchronization therapy at the time of evaluation at a heart failure and cardiac transplantation center. Pacing Clin Electrophysiol. 2010; 33:988-993. [PubMed: 20353413]

13. Mezu U, Ch I, Halder I, London B, Saba S. Women and minorities are less likely to receive an implantable cardioverter defibrillator for primary prevention of sudden cardiac death. Europace. 2012; 14:341-344. [PubMed: 22071382]

14. Hernandez AF, Fonarow GC, Liang L, Al-Khatib SM, Curtis LH, LaBresh KA, Yancy CW, Albert NM, Peterson ED. Sex and racial differences in the use of implantable cardioverter-defibrillators among patients hospitalized with heart failure. JAMA. 2007; 298:1525-1532. [PubMed: 17911497]

15. MacFadden DR, Tu JV, Chong A, Austin PC, Lee DS. Evaluating sex differences in populationbased utilization of implantable cardioverter-defibrillators: role of cardiac conditions and noncardiac comorbidities. Heart Rhythm. 2009; 6:1289-1296. [PubMed: 19695966]

16. Lin G, Meverden RA, Hodge DO, Uslan DZ, Hayes DL, Brady PA. Age and gender trends in implantable cardioverter defibrillator utilization: a population based study. J Interv Card Electrophysiol. 2008; 22:65-70. [PubMed: 18324458]

17. Thomas KL, Al-Khatib SM, Kelsey RC 2nd, Bush H, Brosius L, Velazquez EJ, Peterson ED, Gilliam FR. Racial disparity in the utilization of implantable-cardioverter defibrillators among patients with prior myocardial infarction and an ejection fraction of <or $=35 \%$. Am J Cardiol. 2007; 100:924-929. [PubMed: 17826371]

18. Chan PS, Birkmeyer JD, Krumholz HM, Spertus JA, Nallamothu BK. Racial and gender trends in the use of implantable cardioverter-defibrillators among Medicare beneficiaries between 1997 and 2003. Congest Heart Fail. 2009; 15:51-57. [PubMed: 19379450]

19. Groeneveld PW, Heidenreich PA, Garber AM. Trends in implantable cardioverter-defibrillator racial disparity: the importance of geography. J Am Coll Cardiol. 2005; 45:72-78. [PubMed: 15629377]

20. Matlock DD, Peterson PN, Heidenreich PA, Lucas FL, Malenka DJ, Wang Y, Curtis JP, Kutner JS, Fisher ES, Masoudi FA. Regional variation in the use of implantable cardioverter-defibrillators for primary prevention: results from the National Cardiovascular Data Registry. Circ Cardiovasc Qual Outcomes. 2011; 4:114-121. [PubMed: 21139094]

21. American Hospital Association Data. 2013. http://www.ahadataviewer.com/quickreport/ 
22. Epstein AJ, Polsky D, Yang F, Yang L, Groeneveld PW. Geographic variation in implantable cardioverter-defibrillator use and heart failure survival. Med Care. 2012; 50:10-17. [PubMed: 22167063]

23. Saba S, Ravipati LP, Voigt A. Recent trends in utilization of implantable cardioverter-defibrillators in survivors of cardiac arrest in the United States. Pacing Clin Electrophysiol. 2009; 32:14441449. [PubMed: 19712076]

24. Havmoeller R, Reinier K, Teodorescu C, Uy-Evanado A, Mariani R, Gunson K, Jui J, Chugh SS. Low rate of secondary prevention ICDs in the general population: multiple-year multiple-source surveillance of sudden cardiac death in the Oregon Sudden Unexpected Death Study. J Cardiovasc Electrophysiol. 2013; 24:60-65. [PubMed: 22860692]

25. Miller AL, Wang Y, Curtis J, Masoudi FA, Buxton AE, Wang TY. Optimal medical therapy use among patients receiving implantable cardioverter/defibrillators: insights from the National Cardiovascular Data Registry. Arch Intern Med. 2012; 172:64-67. [PubMed: 22082711]

26. Centers for Medicare \& Medicaid Services. Decision Memo for Implantable Defibrillators. 2005. http://www.cms.gov/medicare-coverage-database/details/nca-decision-memo.aspx? NCAId $=148 \&$ NCDId $=110 \&$ ncdver $=3 \&$ NcaName $=$ Implantable + Defibrillators \&IsPopup=y\&bc=AAAAAAAAEAAA\&

27. Castellanos JM, Smith LM, Varosy PD, Dehlendorf C, Marcus GM. Referring physicians' discordance with the primary prevention implantable cardioverter-defibrillator guidelines: a national survey. Heart Rhythm. 2012; 9:874-881. [PubMed: 22306794] 


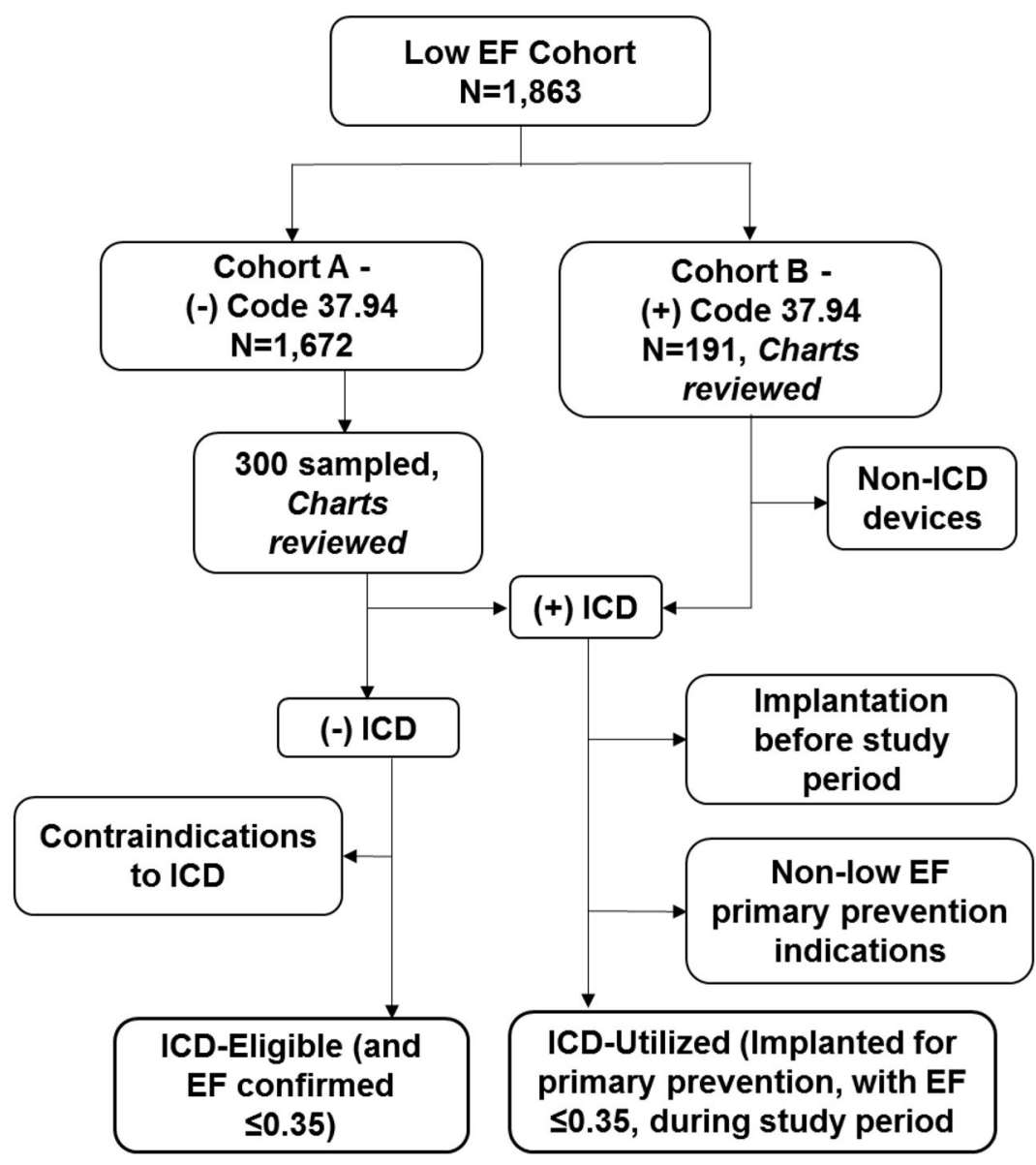

Figure 1.

Process of patient selection and review. 


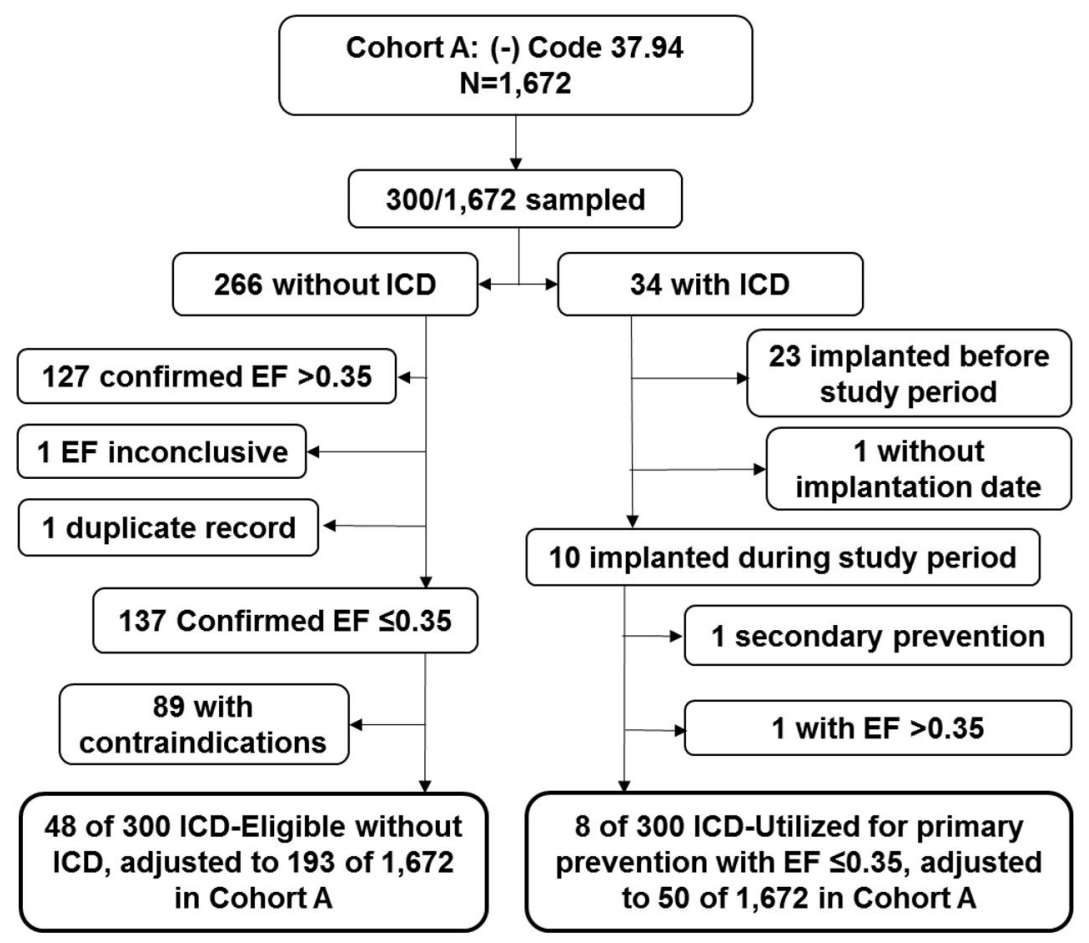

Figure 2.

Flow chart of Cohort A. Sampling correction factors of $8.45\left(C_{1}, D_{1}\right)$ for the private hospital (150 of 1,267 charts sampled) and $2.7\left(C_{2}, D_{2}\right)$ for the county hospital (150 of 405 charts sampled) were applied to estimate ICD-eligible and ICD-utilized populations over the entire cohort. For those with ICD, only those who received ICD during the study period, from June 1, 2011 to May 31, 2012, were selected. 


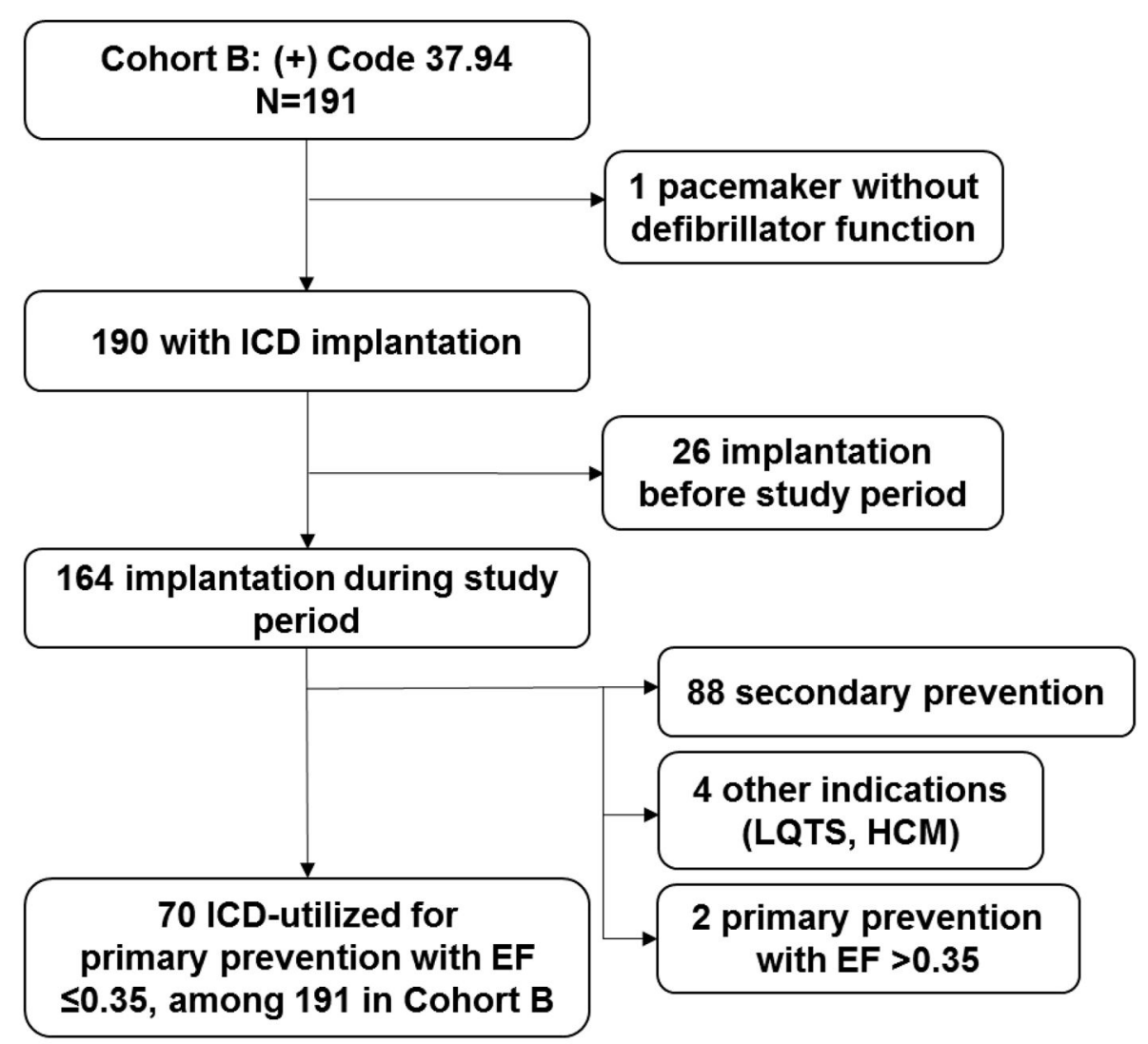

Figure 3.

Flow chart of Cohort B. Those who initially received ICD before the study period were excluded as shown. Seventy patients were found to meet criteria for ICD-utilized for primary prevention with low EF. 


\section{Table 1}

\section{Contraindications for ICD in Cohort A}

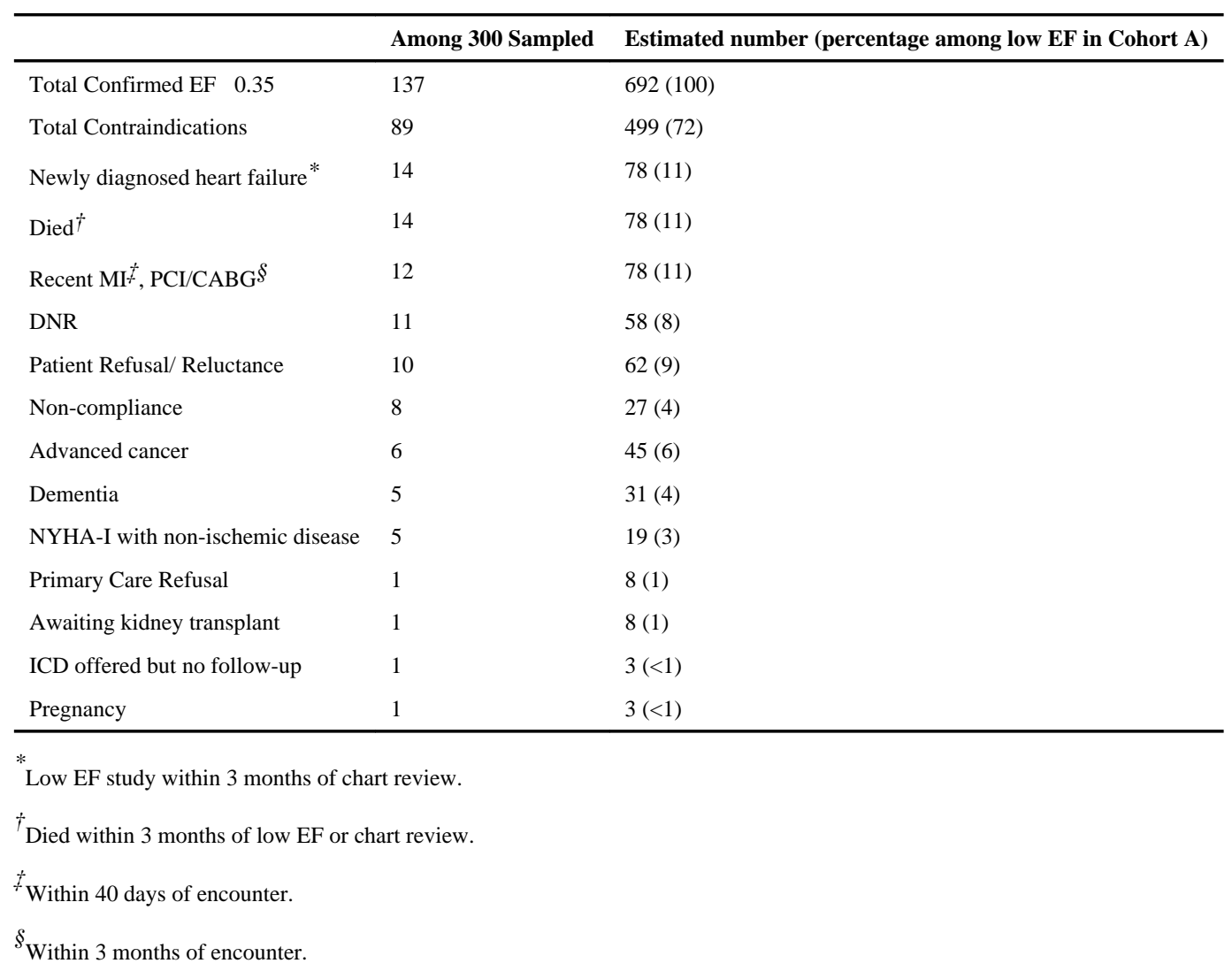

ICD indicates implantable cardioverter-defibrillator; EF, left ventricular ejection fraction; MI, myocardial infarction; PCI, percutaneous coronary intervention; CABG, coronary artery bypass grafting; DNR, do not resuscitate; NYHA, New York Heart Association functional class. 